\title{
Integrated Genomic and Proteomic Analysis of Signaling Pathways in Dendritic Cell Differentiation and Maturation
}

\author{
JOHN RICHARDS,${ }^{a}$ FRANÇOIS LE NAOUR, ${ }^{a}$ SAMIR HANASH, ${ }^{b}$ \\ AND LAURA BERETTA ${ }^{a}$ \\ ${ }^{a}$ Department of Microbiology and Immunology, ${ }^{b}$ Department of Pediatrics, \\ University of Michigan, Ann Arbor, Michigan 48109, USA
}

\begin{abstract}
Dendritic cells (DCs) are antigen-presenting cells that play a major role in initiating primary immune responses. Their phenotypic and functional characteristics are intimately linked to their stage of maturation. The specific biochemical pathways and genes whose expression mediates differentiation of progenitors to DCs and their maturation are largely undefined. We recently utilized two approaches, DNA microarrays and proteomics, to analyze the expression profile of human $\mathrm{CD}_{14}{ }^{+}$blood monocytes and their derived DCs. Approximately $4 \%$ of the genes or proteins expressed were found to be regulated during DC differentiation. Most of these genes were not previously associated with DCs and included genes highly relevant to DC functions (genes involved in antigen presentation, cell adhesion and motility, lipid metabolism). Genes involved in specific signaling pathways, including IKB $\alpha$, PPAR- $\gamma$ and $\mathrm{C} / \mathrm{EBP} \alpha$ as well as two members of the family of transcription factors, interferon regulatory factors (IRFs), were also modified. Modulation of IRF gene expression is of particular interest because of their functional roles in innate and adaptive immune responses. IRF-family members control the expression of proteins that include type-1 interferons, interleukin-12, interleukin-15, MHC molecules and adhesion molecules. They have also been found to play an important role in lymphocyte development. In contrast to DC differentiation, very few genes were modified at the transcript level during DC maturation as determined by microarray experiments. Further analysis suggested that DC maturation is largely controlled by posttranscriptional and posttranslational modifications. The use of proteomics is therefore necessary for a full comprehension of DC maturation process.
\end{abstract}

KEYWORDS: genomic analysis; proteomic analysis; signaling pathways; dendritic cell differentiation; dendritic cell maturation

\section{DENDRITIC CELL DIFFERENTIATION AND MATURATION}

Dendritic cells (DCs) are potent antigen-presenting cells derived from bone marrow that have an integral role in regulating the immune response. ${ }^{1,2}$ The function of DCs correlates directly with their degree of differentiation and maturation. ${ }^{2}$

Address for correspondence: Laura Beretta, Department of Microbiology and Immunology, University of Michigan, 6744 MSII, 1150 West Medical Center Drive, Ann Arbor, Michigan 48109-0620. Voice: 734615 5964; fax: 7346156150.

berettal@umich.edu

Ann. N.Y. Acad. Sci. 975: 91-100 (2002). @ 2002 New York Academy of Sciences. 
Immature DCs are not potent antigen-presenting cells, but continually uptake material from their surroundings by phagocytosis, macropinocytosis, and receptor-mediated endocytosis. ${ }^{2}$ DC maturation leads to the cessation of antigen uptake for antigen presentation. Mature DCs have greater surface levels of MHC, costimulatory, and adhesion molecules making them potent activators of T cells. ${ }^{2}$ Furthermore, mature DCs secrete chemokines such as SLC and MIP-3 $\beta$, which promote the recruitment of naïve and memory T cells. ${ }^{3}$ In vivo, the location of DCs is highly concordant with their function. Immature DCs are located in body tissues were they act as sentinels. ${ }^{4}$ Upon encounter with a pathogen or at a site of inflammation, DCs undergo maturation and migrate through the afferent lymphatics to secondary lymphoid organs where they interact and activate antigen-specific T cells. ${ }^{5}$ Activated T cells leave the secondary lymphoid organs, migrate to the inflammation site, and perform effector functions leading to the clearance of the pathogen. ${ }^{1,2}$

DCs were initially known for their ability to activate T cells. ${ }^{4}$ More recently, the ability of DCs to regulate T cells has become of great interest. A striking example of DC-induced $T$ cell tolerance was observed when influenza matrix peptide (MP)pulsed immature DCs were injected into healthy individuals. ${ }^{6}$ Evaluation of the immune response pre- and post-DC injection demonstrated a decrease in $\mathrm{T}$ cell reactivity against MP. Injection of MP-loaded, mature DCs showed the opposite effect, as T cells had a more robust response towards MP. ${ }^{6,7}$ Determination of whether DCs will be tolerogenic or immunogenic may be related to DC subsets or DC plasticity. An example of how different human DC populations can give different immune responses is demonstrated by comparing human myeloid DCs with human plasmacytoid DCs. ${ }^{8}$ Allogeneic T cell stimulation with myeloid DCs and plasmacytoid DCs results in $\mathrm{T}$ cell secretion of IFN- $\gamma$ and IL-4, IL-5, or IL-10, respectively. Closer analysis of the DCs showed that myeloid DCs secreted IL-12 while plasmacytoid DCs did not. These data along with data obtained in murine models helped develop the idea that tolerance and activation might be induced by different DC subsets. ${ }^{9}$ However, to complicate matters, DC subsets have plasticity in that they exhibit the ability to both activate and tolerize T cells. ${ }^{10}$ The plasticity is associated with the microenvironment where the DC resides. ${ }^{10}$ For example, myeloid DCs cultured in the presence of IL-10 induce T cell tolerance, whereas DCs cultured in the presence of inflammatory stimuli will augment $\mathrm{T}$ cell activation and dsRNA will augment $\mathrm{T}$ cell activation as well as polarize $\mathrm{T}$ cells towards a type 1 response. ${ }^{11,12}$

The ability of DCs to stimulate different immune responses is associated with different states of maturation. ${ }^{2}$ Several microbial products such as dsRNA, LPS, peptidoglycan, and bacterial $\mathrm{CpG}$ islands all seem to stimulate DC maturation. ${ }^{13}$ The capacity of these molecules to stimulate DC maturation is associated with their binding to specific Toll-like receptors present on the DC surface. ${ }^{10}$ Toll-like receptors send signals that result in the activation in NF- $\kappa B$. NF- $\kappa B$ has been associated with the transcriptional regulation of many genes that are detected during DC maturation, including the cytokine IL-12p40 and costimulatory molecules CD80 and CD86. ${ }^{14}$ The common activation of $\mathrm{NF}-\mathrm{\kappa B}$ by toll-like receptors and cytokines does not explain the diversity in DC maturation. For example, DCs matured with peptidoglycans secrete high levels of TNF- $\alpha$, low levels of IL-6 and IL-12, and no IFN- $\alpha$, while poly I:C induces the secretion of IL-12p70, low levels of IFN- $\alpha$, and no TNF- $\alpha$ or IL- 6 after 24 hours incubation. ${ }^{13}$ Individual inflammatory mediators such 
as TNF- $\alpha$, IL-1 $\beta$, IL-6, and prostaglandin E2 (PGE2) have the ability to induce DC maturation. ${ }^{15}$ However, the combinations of inflammatory mediators are synergistic in their ability to induce DC maturation. NF- $\kappa B$ is definitely involved in these responses but what other biochemical pathways and transcription factors are involved in the generation of diversity found in the immune response?

DCs have become accessible for detailed molecular and cell biological analysis and for clinical applications. Microarrays and proteomics are emerging and potentially complementary technologies for identifying the mRNA and protein constituents of living organisms and determining their pattern of expression. ${ }^{16-18}$ Few studies have been undertaken that simultaneously analyzed cell populations at both RNA and protein levels. Potential sources of discordance between RNA and protein levels include translational control and altered protein stability. Additionally, proteomic analysis may uncover posttranslational modifications that are not predictable at the RNA level. Using both oligonucleotide microarrays and proteomics, we performed a global analysis of gene expression during DC differentiation and maturation. ${ }^{19}$

\section{GENOMICS APPLIED TO DENDRITIC CELL DIFFERENTIATION AND MATURATION}

The identification of expressed sequences in a number of species has ushered in the post-genome era. In this era, technologies are becoming available that allow the profiling of tissues and cell populations at the RNA and protein levels. Microarray technology is a promising approach to comparatively analyze genome-wide patterns of mRNA expression, ${ }^{20-27}$ the ultimate goal being to develop arrays that contain every gene in a genome, or selected subsets, against which mRNA expression levels can be quantitatively assessed. DNA microarrays provide an important new tool for the characterization of tissues and cell lines. Large sets of genes involved in signal transduction, differentiation, apoptosis, cell cycle progression, etc., all could be analyzed simultaneously with respect to their expression. Microarray analysis exemplifies the type of comprehensive, large throughput technologies that could yield a molecular based understanding of physiological processes and disease states. There are at present a substantial variety of arrays that may be utilized as well as choices of hardware, software, and multiple sources of DNA for arraying and a variety of protocols for implementation. One technology for cDNA-based microarrays is based on an approach in which cDNA clone inserts are robotically printed onto glass slides and subsequently hybridized to two differentially fluorescently labeled probes to compare the expression patterns of the cells from which they were derived. Another leading technology relies on the use of oligonucleotides. One concept that has been implemented involves the synthesis of tens of thousands of oligonucleotides onto chips using photolithography, as developed by Affymetrix.

Differentiation of $\mathrm{CD}_{1} 4^{+}$blood monocytes into mature DCs can be induced in vitro by treatment with a combination of GM-CSF, IL-4 and TNF- $\alpha .{ }^{10,16}$ Using oligonucleotide arrays, RNA transcript levels for different genes expressed during DC differentiation were determined at day $1\left(\mathrm{CD} 14^{+}\right.$monocytes) and after seven days of GM-CSF/IL-4 treatment (immature DCs) and 14 days of GM-CSF/IL-4 plus TNF- $\alpha$ treatment (mature DCs). Transcripts for approximately $40 \%$ of the 6,300 unique 
genes assessed were detected in all the cell populations tested. We identified a subset of genes which differed in their expression levels during DC differentiation and maturation, by 2.5 -fold or greater.

We identified close to $4 \%$ of the genes and proteins analyzed as regulated during DC differentiation. The regulated genes were in major part related to cell adhesion and motility, growth control, regulation of the immune response and antigen presentation, transcription and signal transduction, and lipid metabolism. Genes known to be differentially expressed during DC differentiation changed their expression accordingly in our analysis. This group included the monocytic marker CD14, CD163 and C5a anaphylatoxin receptor (CD88), which were strongly downregulated, and the cell surface proteins MHC class II, CD1a, CD1b, CD1c, CD36, CD59, CD83, CD86, and CCR7 which were upregregulated with DC differentiation and maturation. The secretion of proteins TARC (CCR4 ligand), MCP-4 and the macrophage-derived chemokine (MDC) was also observed. Most of the 255 regulated genes we uncovered were not previously known to be differentially expressed in DCs.

Interestingly, we identified a large number of genes encoding for proteins involved in cell adhesion and motility which are regulated during DC differentiation. Expression of galectin 2, CD11a/LFA-1 alpha, ninjurin 1, macmarcks, syndecan 2, CD44E, and presenilin 1 was downregulated. Expression of secreted proteins involved in cell motility, autotaxin-t and semaphorin E, reported to play a role in axon guidance in the nervous system, ${ }^{28}$ was upregulated. Therefore, the concomitant decrease in expression of integrins and cell adhesion molecules, the increase in expression of genes involved in cell motility, and regulated expression of enzymes such as alpha 1 antitrypsin and macrophage metalloelastase (HME), likely have an effect on the enhanced migration properties of DCs compared to their precursors.

Differentiation of DCs was accompanied by differential expression of genes involved in the immune response. Noticeable was the upregulation of genes encoding antiinflammatory proteins such as cyclophilin C and TSG-6 ${ }^{29,30}$ with a concomitant decrease in the production of proinflammatory cytokines. Several genes encoding proinflammatory cytokines and their receptors, such as prointerleukin-1- $\beta$, TNF- $\alpha$, CD163, C5a anaphylatoxin receptor, IL-6 receptor, and TNF receptor were downregulated. A noticeable change was the downregulation of a set of chemokines belonging to the IL-8 superfamily such as CTAPIII, MIP2- $\alpha$, MIP2- $\beta$, ENA78, PF4, and IL-8. It has been reported that these chemokines are proinflammatory cytokines that act as potent neutrophil chemoattractants and activators. ${ }^{31}$ Interestingly, these chemokines which were coordinately downregulated have been co-localized to the same genomic region. ${ }^{32}$ Osteopontin, a key cytokine involved in T-lymphocyte activation $^{33}$ was upregulated. The maturation of DCs was accompanied by the upregulation of Mac-2 binding protein. Mac-2 binding protein is an adhesion molecule with a potent immune stimulatory activity. Indeed, it has been demonstrated that Mac-2 binding protein stimulates host defense systems, such as NK and LAK cell activities, and induces the secretion of IL-2. ${ }^{34}$ Upregulation of TGF- $\alpha$ was also observed during DC maturation.

Expression of mRNAs encoding for proteins localized in the nuclear compartment or involved in signaling has been poorly described in DCs. Expression of the interferon regulatory factor 4 (IRF-4), C/EBPa, mrg1, PPARg, TRIP7, SLA, 
Rap1GAP, cAMP-dependent protein kinase, IP3 protein kinase $\mathrm{B}$, cyclophilin $\mathrm{C}$ and cyclins A1, D2, G2 and $\mathrm{H}$ genes, was increased. Expression of IRF-7A, TAL2, NAP-2, EGF-response factor 2, CtBP, IEX-1, SAP49, HRH1, I-кB alpha, Fyb, Net and cyclophilin $\mathrm{F}$ genes was decreased. Of these genes, IRF-4 and IRF-7 are of particular interest. In IRF-4 ${ }^{-/-}$mice, there are severe immunological defects in $\mathrm{T}$ and $\mathrm{B}$ cell function. ${ }^{35}$ Antigen-presenting cells appeared normal as $\mathrm{T}$ cell lines recognized processed antigen. However, these mice were unable to mount a primary immune response against an allogeneic tumor, suggesting a defect in DC function. The role of IRF-7 has been traditionally involved in the activation of type 1 interferons. ${ }^{36,37}$ More recently, IRF-7 was shown to be involved in the differentiation of monocytes into macrophages. ${ }^{38}$ The decrease in IRF-7 during DC differentiation versus increased expression in macrophage differentiation may suggest a critical role for IRF-7 at the crossroad between macrophage and DC differentiation from monocytes.

Finally, the regulation of a large group of genes encoding for lipid-binding proteins or enzymes involved in lipid metabolism was observed in DC differentiation. Several genes encoding enzymes or proteins involved in the production, uptake, transport, and solubilization of cholesterol and fatty acids were upregulated in DCs. This group includes apolipoprotein E, apolipoprotein C-I, ABCG1, lysosomal acid lipase, and lipoprotein lipase. The fatty acids are translocated from the extracellular environment to the cytoplasm by the fatty acid translocase (FAT/CD36) and then solubilized and transported by fatty acid binding proteins (FABPs) to the site where they are metabolized. ${ }^{39}$ We reported marked upregulation of CD36 as well as of the lipid binding proteins FABP3, FABP4, FABP5, CRABPII, and ACBP. Upregulation of FABPs was concomitant with a strong downregulation of the S100 proteins, MRP8 and MRP14. Interestingly, MRP8 and MRP14 are expressed by myeloid cells during inflammatory reactions, and it has been reported that MRP-8/MRP-14 heterodimer (FA-p34) has a fatty acid binding activity and specifically binds (poly)unsaturated fatty acids. ${ }^{40-42}$ In contrast, FABP4 and FABP5 bind saturated or (mono) unsaturated fatty acids with a high affinity. Long-chain fatty acids and acylCoA esters affect a large number of cell functions during cell growth and differentiation, including signal transduction, gene regulation, ion channel activities, and membrane fusion. ${ }^{39,43}$ In this context, we observed an upregulation of 15-lipoxygenase which promotes the formation of lipoxins that are modulators of leukocyte recruitment. ${ }^{44-46}$

\section{PROTEOMICS APPLIED TO DENDRITIC CELL DIFFERENTIATION AND MATURATION}

Proteins are the most functional component encoded for in the genome. The large-scale profiling of gene expression at the protein level has a long history that predates profiling at the RNA level. ${ }^{47}$ In the past decade, mass spectrometry has provided the means for the identification, through sequence database searching, of proteins separated by two-dimensional electrophoresis or other means, at an unprecedented level of sensitivity and speed. ${ }^{48}$ The availability of a full set of human and other mammalian sequences of expressed genes and the emergence of mass spectrometry as an important tool for protein identification have led to a resurgence of 
interest in protein analysis. Critical to protein investigations has been the availability of suitable technology for the large-scale analysis of complex protein mixtures that provides high speed and automation as well as high sensitivity. A number of technologies have been investigated or are under development. However it is generally agreed that at the present time, 2D-PAGE remains the "gold standard" for resolving large protein mixtures.

To identify protein changes during the differentiation and maturation of the monocyte-derived DCs, total proteins were extracted as described for the microarray analysis. Following protein separation by two-dimensional gel electrophoresis, gels were silver stained and digitized. 2-D protein patterns were matched by computer analysis; 900 protein spots were matched and quantitated. Whereas the overall 2-D patterns of $\mathrm{CD}_{1} 4^{+}$monocytes, immature and mature DCs were largely similar, numerous protein changes were reproducibly detected. As for the microarray analysis, we selected protein spots whose intensities changed in all experiments by 2.5 fold or greater during DC differentiation or maturation. A set of 37 proteins was identified.

Of the 37 spots excised from the gels, 18 were identified without ambiguity, by mass spectrometry. Specific antibodies confirmed the identification based on mass spectrometry for all proteins analyzed by Western-blotting. The proteins identified

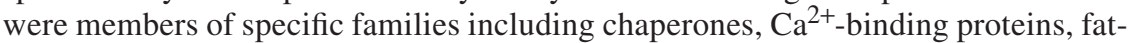
ty acid binding proteins and structural proteins. Expression of three members of the fatty acid binding protein (FABP) family, FABP4, FABP5 and Acyl-CoA-binding protein, (ACBP) was highly increased after seven days of culture. The increased protein and RNA levels for these genes were concordant. Concomitant with the upregulation of FABP4 and FABP5, we observed a strong downregulation of two members of the S100 family, the myeloid-related proteins MRP14 and MRP8. Interestingly, it has been recently shown that the heterodimer MRP8/MRP14, designated fatty-acid p34 (FA-p34), exerts a fatty-acid binding activity. ${ }^{40-42}$ MRP14 and MRP8 downregulation was progressive upon DC differentiation and maturation, leading to a 9- and 12 -fold decrease in spot intensities, respectively. Again, the results obtained for these two genes at both the RNA and protein levels were highly concordant.

Several proteins known for their chaperone activity including hsp73, hsp27, and calreticulin were also regulated during DC differentiation. An emerging hypothesis is that heat shock proteins participate in antigen processing and presentation and play a central role in the activation of T lymphocytes by DCs. ${ }^{49-51}$ Hsp70 targets immature DC precursors to enhance antigen uptake. ${ }^{52}$ We observed an upregulation of hsp73 protein, related to the hsp70 family, ${ }^{53}$ during DC differentiation. Hsp73 has been recently reported to bind specifically to the cell surface of monocytic and dendritic cell lines and to be internalized spontaneously by receptor-mediated endocytosis. ${ }^{50}$ In addition, the murine hsp73 has been recently reported to accumulate in exosomes from immatures DCs. ${ }^{54}$ The role of hsp27 upregulation during DC differentiation is less clear. It has been reported that an increase in cellular levels of hsp27 promotes a resistance of monocytes to apoptotic cell death. ${ }^{55,56}$ Increased hsp27 expression in DCs may therefore have a protective role against cytotoxicity. In contrast to hsp27 and hsp73, the cognate chaperone protein calreticulin was downregulated during DC differentiation owing to posttranslational modification. In addition, whereas the expression of hsp27 and hsp73 was maximal in immature DCs, 
calreticulin was mostly downregulated during DC maturation. Calreticulin participates in the assembly of MHC class I with peptide and beta 2-microglobulin in the endoplasmic reticulum, a process required for the presentation of antigenic peptides to CTLs at the cell surface. ${ }^{57,58}$ In addition, it has been recently reported that calreticulin elicits tumor- and peptide-specific immunity. ${ }^{59}$ Calreticulin displays in vivo peptide-binding activity and can elicit CTL responses against bound peptides. ${ }^{60}$

There were discrepancies between the protein and gene expression data for vimentin, hsp27, and calreticulin. Close analysis of the microarray hybridization data showed saturation level intensities for vimentin and hsp resulting from their high-level expression. Therefore, the discordance between mRNA and protein levels observed in our data for these genes most likely reflects their high expression level, reaching saturation at the RNA hybridization level using microarrays, but not at the protein level using 2-D gels. The calreticulin protein was found to be downregulated with DC differentiation in our 2-D gel analysis, whereas the corresponding transcript was unchanged at the RNA level by microarray analysis. Hybridization data for calreticulin transcript did not show any saturation. Interestingly, a protein with an estimated MW of $32 \mathrm{kDa}$ and PI of 4.1, was found to be induced in immature DCs. After enzymatic digestions using trypsin or endoproteinase Lys-C and analysis of the resulting peptides by MALDI-TOF mass spectrometry, the peptide masses were consistent with those of peptides derived from calreticulin, a protein with a MW of $48 \mathrm{kDa}$ and a PI of 4.3. Altogether, proteomic analysis allowed us to identify a truncated form of calreticulin, present only in DCs. We designated this novel form of calreticulin as $\mathrm{Crt32}$. This form contains the P-domain, a site of chaperone activity, and the $\mathrm{C}$-domain, which contains the endoplasmic reticulum retrieval sequence, but lacks the $\mathrm{N}$-domain. A calreticulin fragment corresponding to the $\mathrm{N}$-domain has been recently purified from the supernatant of an EBV-immortalized cell line. This fragment, named vasostatin, is an angiogenesis inhibitor that exerts antitumor effects in vivo. ${ }^{61,62}$ Therefore, even though the $\mathrm{C}$-terminal end of vasostatin has not been precisely characterized, Crt32 most likely corresponds to the complementary part of vasostatin, following cleavage of calreticulin. A decrease in levels of the cognate form of calreticulin and an increase in Crt32 levels may be relevant to DC function and the precise function(s) of Crt32 in mature DCs is currently under investigation. Therefore, whereas microarray analysis did not show any changes involving calreticulin, proteomic analysis allowed the detection of a posttranslational modification of calreticulin occurring during DC differentiation.

\section{FINAL COMMENTS}

DCs acquire their function with differentiation and maturation, which occurs through a programmed expression of specific proteins. The combination of both genomics relying on the quantitative analysis of mRNAs, and proteomics, relying on quantitative analysis and identification of proteins, demonstrated a good correlation between transcripts and proteins during DC differentiation, but not during DC maturation. Therefore complete understanding of the mechanisms underlying DC maturation would necessitate further proteomic analysis of specific subcellular frac-

tions of the cell. Indeed, proteins represent the most functional compartment of a 
cell, and the information obtained at the protein level cannot simply be predicted from examining expression at the RNA level. The proteomics approach is also appropriate to identify posttranslational modifications, which may regulate protein function. An example is that during DC differentiation MHC class II molecules are synthesized but are not stably expressed on the cell surface. ${ }^{63}$ However, fully mature DCs stably express MHC class II molecules on the cell surface while biosynthetic and endocytic traffic ceases. ${ }^{63}$

The oligonucleotide array and proteomics analyses have uncovered novel genes and proteins with potential roles in DC function, differentiation and/or maturation. Microarray analysis has identified important changes in genes involved in cell adhesion and motility, immune response, and growth control as well as in lipid metabolism. Following the simultaneous analysis of several thousand genes at the mRNA level, the challenge is to efficiently utilize this information to develop a better understanding of DC function. This study also demonstrates that a proteomics approach may provide information that could not be obtained at the RNA level, owing either to poor correlation between mRNA and protein levels or to posttranslational modifications that may result in several isoforms generated from one mRNA, as in the case of calreticulin in our study. Genes and proteins identified to be selectively expressed in DCs, may provide further understanding of the biological function of DCs in host defense systems and of the mechanisms of antigen processing and presentation.

\section{REFERENCES}

1. Banchereau, J. et al. 2000. Immunobiology of dendritic cells. Annu. Rev. Immunol. 18: 767-811.

2. Banchereau, J. \& R.M. Steinman. 1998. Dendritic cells and the control of immunity. Nature 392: 245-252.

3. Cyster, J.G. 1999. Chemokines and cell migration in secondary lymphoid organs. Science 286: 2098-2102.

4. Steinman, R.M. 1991. The dendritic cell system and its role in immunogenicity. Annu. Rev. Immunol. 9: 271-296.

5. Steinman, R.M. 2001. Dendritic cells and the control of immunity: enhancing the efficiency of antigen presentation. Mt. Sinai J. Med. 68: 106-166.

6. DHODAPKAR, M.V. et al. 2001. Antigen-specific inhibition of effector T cell function in humans after injection of immature dendritic cells. J. Exp. Med. 193: 233-238.

7. DHODAPKAR, M.V. et al. 1999. Rapid generation of broad T-cell immunity in humans after a single injection of mature dendritic cells. J. Clin. Invest. 104: 173-180.

8. Rissoan, M.C. et al. 1999. Reciprocal control of T helper cell and dendritic cell differentiation [see comments]. Science 283: 1183-1186.

9. Maldonado-Lopez, R. \& M. Moser. 2001. Dendritic cell subsets and the regulation of Th1/Th2 responses. Semin. Immunol. 13: 275-282.

10. Lanzavecchia, A. \& F. Sallusto. 2001. The instructive role of dendritic cells on T cell responses: lineages, plasticity and kinetics. Curr. Opin. Immunol. 13: 291-298.

11. Steinbrink, K. et al. 1997. Induction of tolerance by IL-10-treated dendritic cells. J. Immunol. 159: 4772-4780.

12. Cella, M. et al. 1999. Maturation, activation, and protection of dendritic cells induced by double-stranded RNA. J. Exp. Med. 189: 821-829.

13. KADOWAKI, N. et al. 2001. Subsets of human dendritic cell precursors express different toll-like receptors and respond to different microbial antigens. J. Exp. Med. 194: 863-869.

14. Hoffmann, J.A. et al. 1999. Phylogenetic perspectives in innate immunity. Science 284: $1313-1318$. 
15. JonUleit, H. et al. 1997. Pro-inflammatory cytokines and prostaglandins induce maturation of potent immunostimulatory dendritic cells under fetal calf serum-free conditions. Eur. J. Immunol. 27: 3135-3142.

16. Lockhart, D.J. \& E.A. WinZeler. 2000. Genomics, gene expression and DNA arrays. Nature 405: 827-836.

17. Hanash, S.M. 1998. Two-dimensional Gel Electrophoresis. In Gel Electrophoresis of Proteins: A Practical Approach. B.D. Hames \& D. Rickwood, Eds.: 189-211. Oxford University Press. Oxford.

18. Pandey, A. \& M. Mann. 2000. Proteomics to study genes and genomes. Nature 405: 837-846.

19. Le Naour, F. et al. 2001. Profiling changes in gene expression during differentiation and maturation of monocyte-derived dendritic cells using both oligonucleotide microarrays and proteomics. J. Biol. Chem. 276: 17920-17931.

20. Chen, Y., E. Dougherty \& M. Bittner. 1997. Ratio-based decisions and the quantitative analysis of cDNA micro-array images. J. Biomed. Optics 2: 365.

21. Brown, P.O. \& D. Botstein. 1999. Exploring the new world of the genome with DNA microarrays. Nat. Genet. 21: 33-37.

22. DeRISI, J. et al. 1996. Use of a cDNA microarray to analyse gene expression patterns in human cancer. Nat. Genet. 14: 457-460.

23. KHAn, J. et al. 1998. Gene expression profiling of alveolar rhabdomyosarcoma with cDNA microarrays. Cancer Res. 58: 5009-5013.

24. SchenA, M. 1996. Genome analysis with gene expression microarrays. Bioessays 18: 427-431.

25. Schena, M. et al. 1995. Quantitative monitoring of gene expression patterns with a complementary DNA microarray. Science 270: 467-470.

26. Schena, M. et al. 1996. Parallel human genome analysis: microarray-based expression monitoring of 1000 genes. Proc. Natl. Acad. Sci. USA 93: 10614-10619.

27. TRENT, J.M. et al. 1997. Use of microgenomic technology for analysis of alterations in DNA copy number and gene expression in malignant melanoma. Clin. Exp. Immunol. 107(Suppl. 1): 33-40.

28. Bagnard, D. et al. 1998. Semaphorins act as attractive and repulsive guidance signals during the development of cortical projections. Development 125: 5043-5053.

29. Trahey, M. \& I.L. Weissman. 1999. Cyclophilin C-associated protein: a normal secreted glycoprotein that down-modulates endotoxin and proinflammatory responses in vivo. Proc. Natl. Acad. Sci. USA 96: 3006-3011.

30. WisNIEWSKI, H.G. \& J. VILCEK. 1997. TSG-6: an IL-1/TNF-inducible protein with anti-inflammatory activity. Cytokine Growth Factor Rev. 8: 143-156.

31. Baggiolini, M., P. Loetscher \& B. Moser. 1995. Interleukin-8 and the chemokine family. Int. J. Immunopharmacol. 17: 103-108.

32. O’Donovan, N., M. Galvin \& J.G. Morgan. 1999. Physical mapping of the CXC chemokine locus on human chromosome 4. Cytogenet. Cell Genet. 84: 39-42.

33. AshKar, S. et al. 2000. Eta-1 (osteopontin): an early component of type-1 (cell-mediated) immunity. Science 287: 860-864.

34. Ullrich, A. et al. 1994. The secreted tumor-associated antigen $90 \mathrm{~K}$ is a potent immune stimulator. J. Biol. Chem. 269: 18401-18407.

35. MitTRUCKER, H.W. et al. 1997. Requirement for the transcription factor LSIRF/IRF4 for mature B and T lymphocyte function. Science 275: 540-543.

36. TANiguCHI, T. et al. 2001. IRF family of transcription factors as regulators of host defense. Annu. Rev. Immunol. 19: 623-655.

37. Mamane, Y. et al. 1999. Interferon regulatory factors: the next generation. Gene 237: $1-14$.

38. Lu, R. \& P.M. Pitha. 2001. Monocyte differentiation to macrophage requires interferon regulatory factor 7. J. Biol. Chem. 276: 45491-45496.

39. Glatz, J.F. \& G.J. van DeR Vusse. 1996. Cellular fatty acid-binding proteins: their function and physiological significance. Prog. Lipid Res. 35: 243-282.

40. Klempt, M. et al. 1997. The heterodimer of the Ca2+-binding proteins MRP8 and MRP14 binds to arachidonic acid. FEBS Lett. 408: 81-84. 
41. KerkHoff, C. et al. 1999. The two calcium-binding proteins, S100A8 and S100A9, are involved in the metabolism of arachidonic acid in human neutrophils. J. Biol. Chem. 274: 32672-32679.

42. Siegenthaler, G. et al. 1997. A heterocomplex formed by the calcium-binding proteins MRP8 (S100A8) and MRP14 (S100A9) binds unsaturated fatty acids with high affinity. J. Biol. Chem. 272: 9371-9377.

43. Faergeman, N.J. \& J. KnUdSEn. 1997. Role of long-chain fatty acyl-CoA esters in the regulation of metabolism and in cell signalling. Biochem. J. 323(Pt. 1): 1-12.

44. Clarkson, M.R. et al. 1998. Leukotrienes and lipoxins: lipoxygenase-derived modulators of leukocyte recruitment and vascular tone in glomerulonephritis. Nephrol. Dial. Transplant. 13: 3043-3051.

45. Kuhn, H. \& B.J. Thiele. 1995. Arachidonate 15-lipoxygenase. J. Lipid Mediat. Cell Signal. 12: 157-170.

46. Sigal, E. \& D.J. ConRad. 1994. Human 15-lipoxygenase: a potential effector molecule for interleukin-4. Adv. Prostaglandin Thromboxane Leukotriene Res. 22: 309 316.

47. Hanash, S.M. 2000. Biomedical applications of two-dimensional electrophoresis using immobilized pH gradients: current status. Electrophoresis 21: 1202-1209.

48. Patterson, S.D. 2000. Mass spectrometry and proteomics. Physiol. Genomics 2: 59-65.

49. Colaco, C.A. 1998. Towards a unified theory of immunity: dendritic cells, stress proteins and antigen capture. Cell. Mol. Biol. (Noisy-le-grand) 44: 883-890.

50. ARNOLD-SCHILD, D. et al. 1999. Cutting edge: receptor-mediated endocytosis of heat shock proteins by professional antigen-presenting cells. J. Immunol. 162: 37573760 .

51. Manara, G.C. et al. 1993. New insights suggesting a possible role of a heat shock protein $70-\mathrm{kD}$ family-related protein in antigen processing/presentation phenomenon in humans. Blood 82: 2865-2871.

52. TODRYK, S. et al. 1999. Heat shock protein 70 induced during tumor cell killing induces Th1 cytokines and targets immature dendritic cell precursors to enhance antigen uptake. J. Immunol. 163: 1398-1408.

53. Dworniczak, B. \& M.E. Mirault. 1987. Structure and expression of a human gene coding for a $71 \mathrm{kd}$ heat shock 'cognate' protein. Nucleic Acids Res. 15: 5181-5197.

54. THERY, C. et al. 1999. Molecular characterization of dendritic cell-derived exosomes. Selective accumulation of the heat shock protein hsc73. J. Cell Biol. 147: 599-610.

55. JaAttela, M. \& D. Wissing. 1993. Heat-shock proteins protect cells from monocyte cytotoxicity: possible mechanism of self-protection. J. Exp. Med. 177: 231-236.

56. Samali, A. \& T.G. CotTER. 1996. Heat shock proteins increase resistance to apoptosis. Exp. Cell Res. 223: 163-170.

57. MichalaK, M. et al. 1999. Calreticulin: one protein, one gene, many functions. Biochem. J. 344 (Pt. 2): 281-292.

58. Krause, K.H. \& M. MichalaK. 1997. Calreticulin. Cell 88: 439-443.

59. Basu, S. \& P.K. SRIvastava. 1999. Calreticulin, a peptide-binding chaperone of the endoplasmic reticulum, elicits tumor- and peptide-specific immunity. J. Exp. Med. 189: 797-802.

60. NAIR, S. et al. 1999. Calreticulin displays in vivo peptide-binding activity and can elicit CTL responses against bound peptides. J. Immunol. 162: 6426-6432.

61. PIKE, S.E. et al. 1998. Vasostatin, a calreticulin fragment, inhibits angiogenesis and suppresses tumor growth. J. Exp. Med. 188: 2349-2356.

62. Pike, S.E. et al. 1999. Calreticulin and calreticulin fragments are endothelial cell inhibitors that suppress tumor growth. Blood 94: 2461-2468.

63. WatTs, C. 1997. Immunology. Inside the gearbox of the dendritic cell. Nature 388: 724-725. 